\title{
Non-existence of planar projective Stewart Gough platforms with elliptic self-motions
}

\author{
Georg Nawratil
}

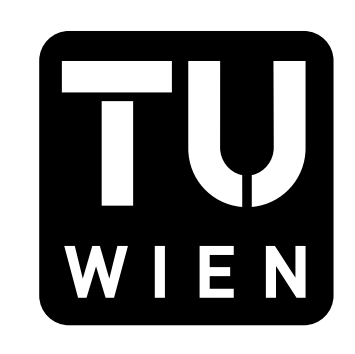

Institute of Discrete Mathematics and Geometry

Research was supported by FWF (I 408-N13)

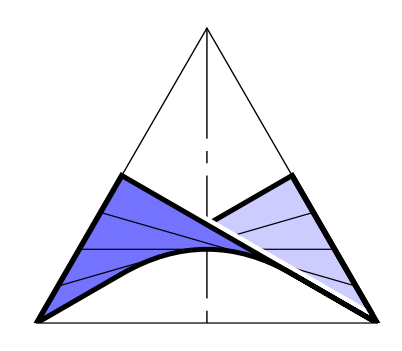




\section{Overview}

1. Introduction

2. Elliptic self-motions

3. Historical results

4. Proof of the Conjecture

5. Conclusion

6. References 


\section{What is a self-motion of a SGP?}

The geometry of a SGP is given by the six base anchor points $M_{i} \in \Sigma_{0}$ and by the six platform points $\mathrm{m}_{i} \in \Sigma$ for $i=1, \ldots, 6$.

A SGP is called planar, if $M_{1}, \ldots, M_{6}$ are coplanar and $\mathrm{m}_{1}, \ldots, \mathrm{m}_{6}$ are coplanar. The carrier planes are denoted by $\pi_{\mathrm{M}}$ resp. $\pi_{\mathrm{m}}$.

$\mathrm{M}_{i}$ and $\mathrm{m}_{i}$ are connected with a $\mathrm{S} \underline{\mathrm{S}}$ leg.

If all $\underline{P}$-joints are locked, a SGP is in general rigid. But, under particular conditions, the manipulator can perform an $n$-parametric motion $(n>0)$, which is called self-motion.

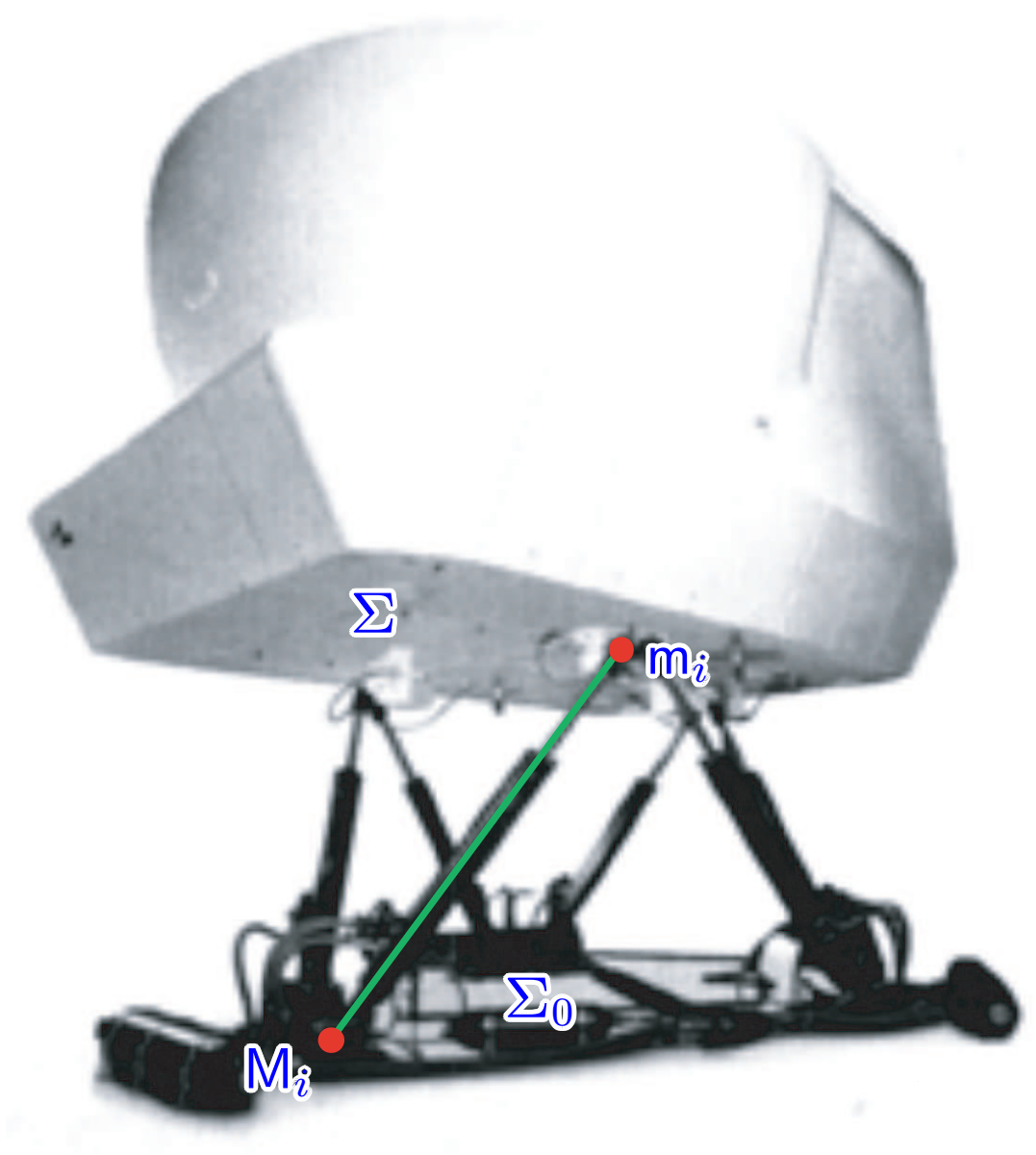




\section{Planar projective SGPs}

\section{Definition 1}

A planar SGP is called projective, if $\mathrm{M}_{i}$ and $\mathrm{m}_{i}$ are related by a non-singular projectivity $\kappa: \mathrm{m}_{i} \mapsto \mathrm{M}_{i}$ for $i=1, \ldots, 6$.

Theorem 1 Merlet [1]

A SGP is singular (infinitesimal flexible, shaky), if and only if, the carrier lines of the six SPS legs belong to a linear line complex.

A planar projective SGP is singular in every possible configuration (= architecturally singular), if and only if, one set of anchor points is located on a conic section (e.g. Chasles [2], Röschel and Mick [3], Karger [4]).

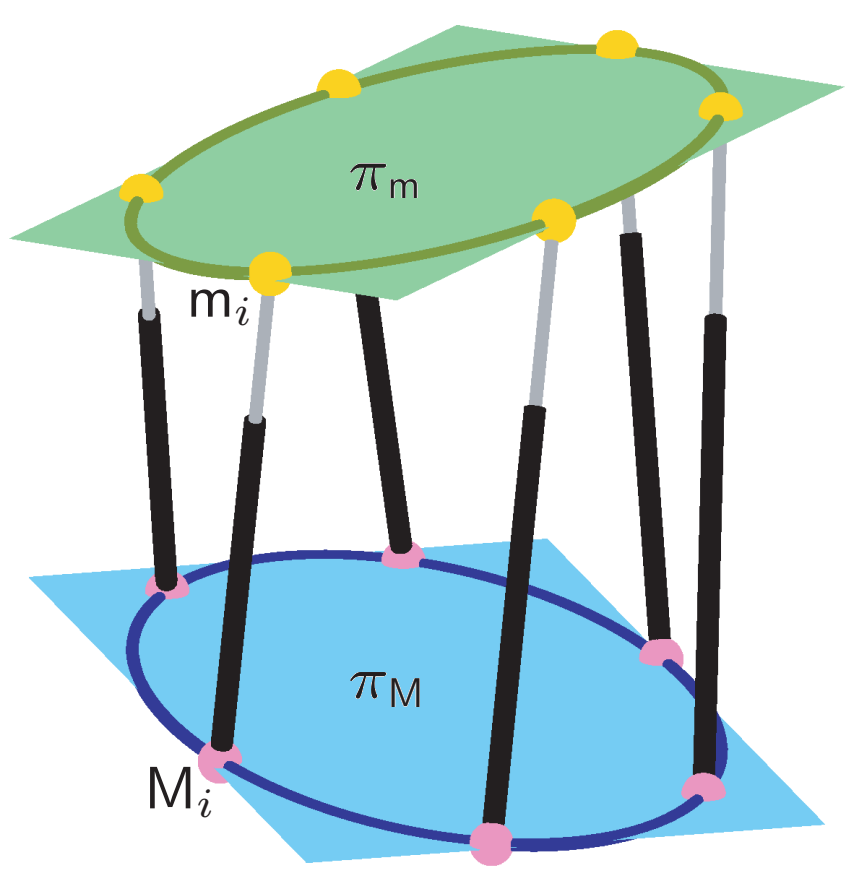




\section{Related results}

As architecturally singular SGPs are redundant, they possess self-motions in each pose (over $\mathbb{C})$.

Therefore we are only interested in non-architecturally singular planar projective SGPs with self-motions.

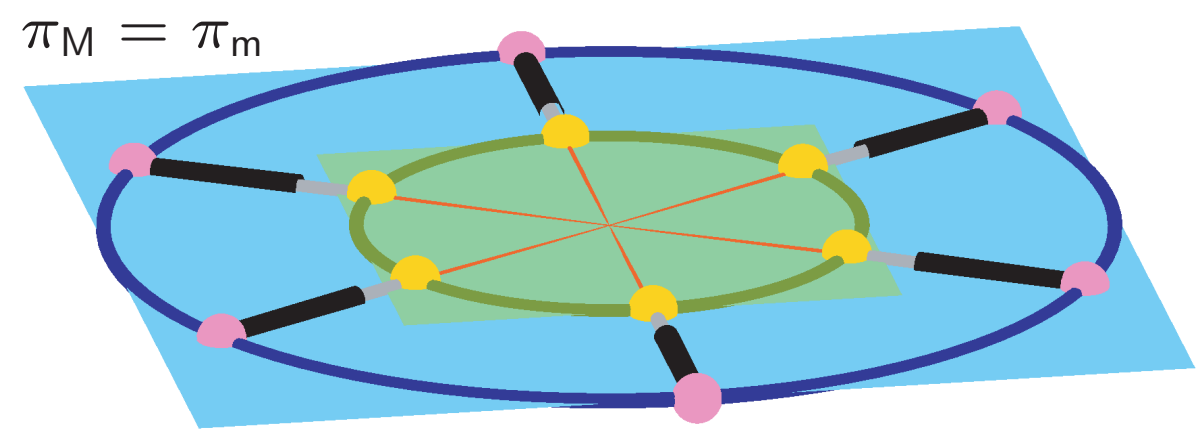

Lemma 1 Nawratil [5]

A two-parametric set of additional legs $\overline{\mathrm{mM}}$ with $\mathrm{m} \kappa=\mathrm{M}$ can be attached to non-architecturally singular planar projective SGPs without changing the direct kinematics and singularity surface.

Moreover it was shown in [5] that non-architecturally singular planar projective SGPs can either have pure translational self-motions or elliptic self-motions. 


\section{Elliptic self-motions}

As the geometry of all manipulators with translational self-motions was already determined in [5], we focused on the study of elliptic self-motions, which can be defined as follows, if we take into account that $s$ denotes the line of intersection of $\pi_{\mathrm{M}}$ and $\pi_{\mathrm{m}}$ in the projective extension of the Euclidean 3-space:

\section{Definition 2}

A self-motion of a non-architecturally singular planar projective SGP is called elliptic, if in each pose of this motion $s$ exists with $s=s \kappa$ and where the projectivity from $s$ onto itself is elliptic.

Moreover we can assume that $\mathrm{s}$ is not the ideal line, as otherwise we get an affine planar SGP, which was already discussed in [5].

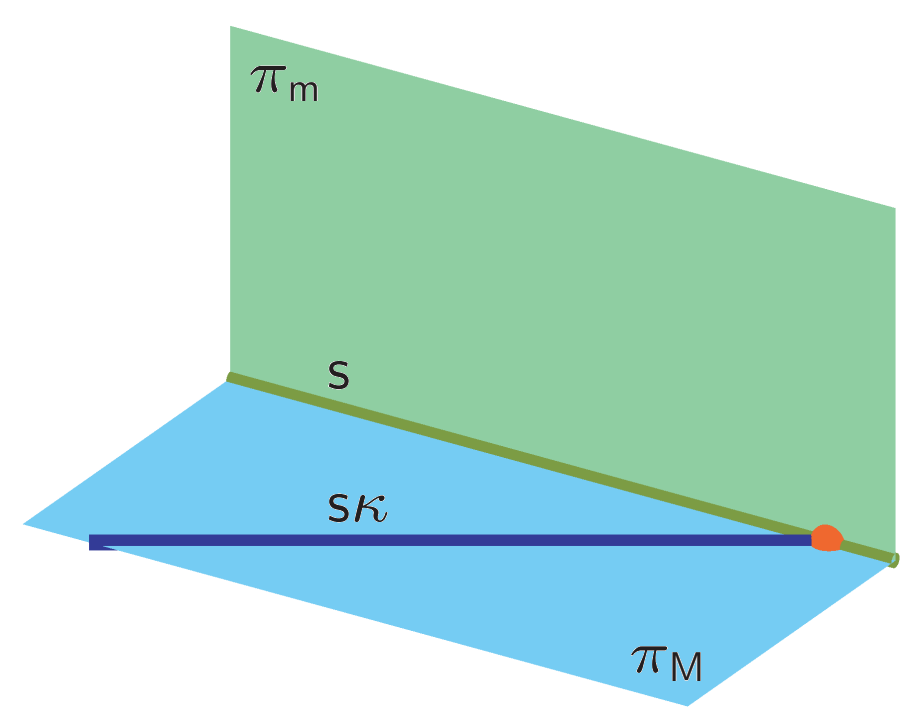




\section{Results on Elliptic self-motions}

It remains open, whether elliptic self-motions even exist, as no example is known. In the case of existence the following theorem has to hold:

\section{Theorem 2 Nawratil [6]}

1. A non-architecturally singular planar projective SGP possesses in each pose of an elliptic self-motion exactly two instantaneous dofs.

2. Elliptic self-motions have to be one-parametric motions.

Moreover the angle $\gamma$ enclosed by the unique pair of ideal points $(f, F)$ with $f \kappa=F$ has to remain constant during the self-motion of a planar projective SGP.

Theorem 3 Nawratil [6]

There does not exist a non-architecturally singular planar projective SGP with an orthogonal elliptic self-motion $\left(\Leftrightarrow \gamma=90^{\circ}\right)$. 


\section{Conjecture}

\section{Conjecture}

Non-architecturally singular planar projective SGPs with an elliptic self-motion do not exist.

We tried to prove this conjecture analogously to Theorem 3, whose proof was done analytically.

Indeed the problem under consideration has only one more unknown, namely the angle $\gamma$, but exactly this additional variable effects the computational complexity enormously. In this case we were not able to solve the problem analytically, due to its high degree of non-linearity.

Therefore we have to come up with another idea for proving this conjecture. This new approach is a purely geometric one, which is based on the following old geometric/kinematic results. 


\section{Elementary facts on regular ruled quadrics}

The one-sheeted hyperboloid and the hyperbolic paraboloid carry two sets of generators (regulus $\mathcal{R}$ and associated regulus $\mathcal{R}^{\times}$). All lines within one set are skew to each other and each line of one set is intersected by all lines of the other set. $\Longrightarrow$ Regular ruled quadric is uniquely determined by three pairwise skew generators
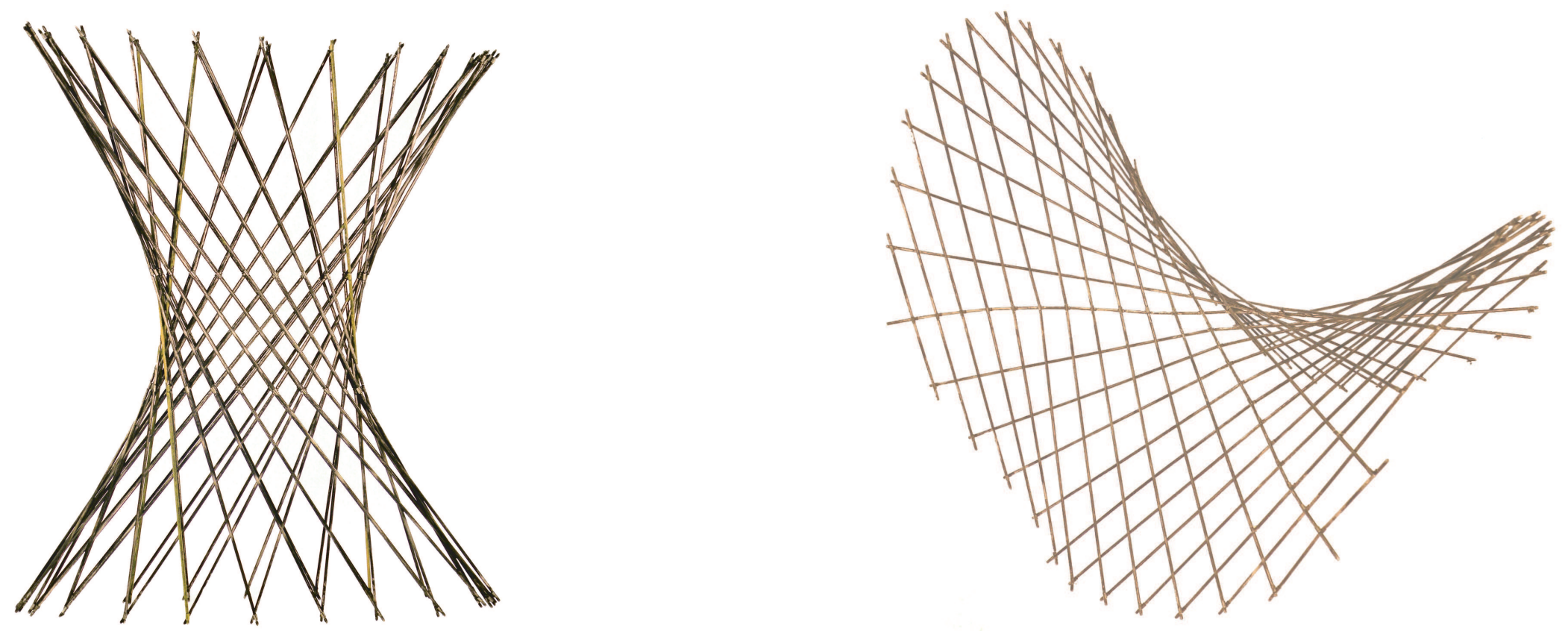


\section{Historical results}

In 1873, the following theorem was given by Henrici [7]:

\section{Theorem 4}

If the generators of a hyperboloid $\Phi$ of one sheet are constructed of rods, jointed at the points of crossing in a way that at each intersection point one rod is free movable about the other one, then the surface is not rigid, but permits a deformation into a one-parametric set $\mathcal{H}$ of hyperboloids.

In 1899, Schur [8] presented a very elegant proof for Henrici's theorem, which also showed that this theorem remains valid if the one-sheeted hyperboloid is replaced by a hyperbolic paraboloid.

Remark: Based on these results, Wiener [9] made some deformable models of one-sheeted hyperboloids and hyperbolic paraboloids. 


\section{Historical results}

The following theorem is well known (cf. page 222 of Koenigs [10]):

\section{Theorem 5}

If three points $\mathrm{m}_{1}, \mathrm{~m}_{2}, \mathrm{~m}_{3}$ of a line $\mathrm{g}$ run on spheres, where the centers $M_{1}, M_{2}, M_{3}$ are also located on a line $\mathrm{G}$, then every point $\mathrm{m}$ of $\mathrm{g}$ has a spherical trajectory, where the center $M$ of this sphere belongs to $G$ and fulfills the relation:

$$
C R\left(\mathrm{~m}_{1}, \mathrm{~m}_{2}, \mathrm{~m}_{3}, \mathrm{~m}\right)=C R\left(\mathrm{M}_{1}, \mathrm{M}_{2}, \mathrm{M}_{3}, \mathrm{M}\right) \text {, }
$$

where $C R$ denotes the cross-ratio.

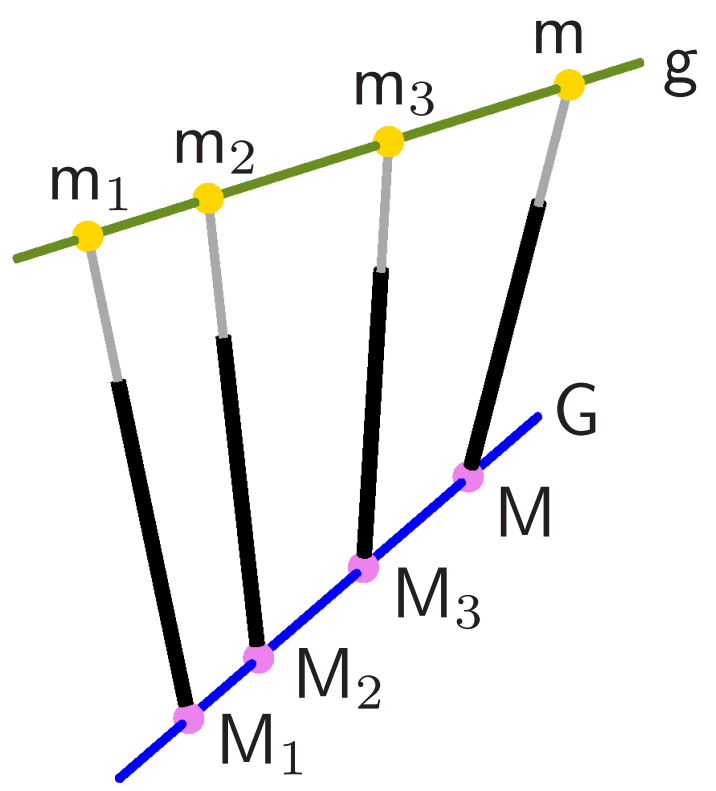

Moreover it is a well known fact of projective geometry that the one-parametric set of lines $[\mathrm{m}, \mathrm{M}]$ with $\mathrm{m}$ and $\mathrm{M}$ of Theorem 5 span a regulus $\mathcal{R}$ of a regular ruled quadric, if $g$ and $G$ are skew and $m_{1}, m_{2}, m_{3}$ and $M_{1}, M_{2}, M_{3}$ are pairwise distinct. 


\section{Proof of the Conjecture}

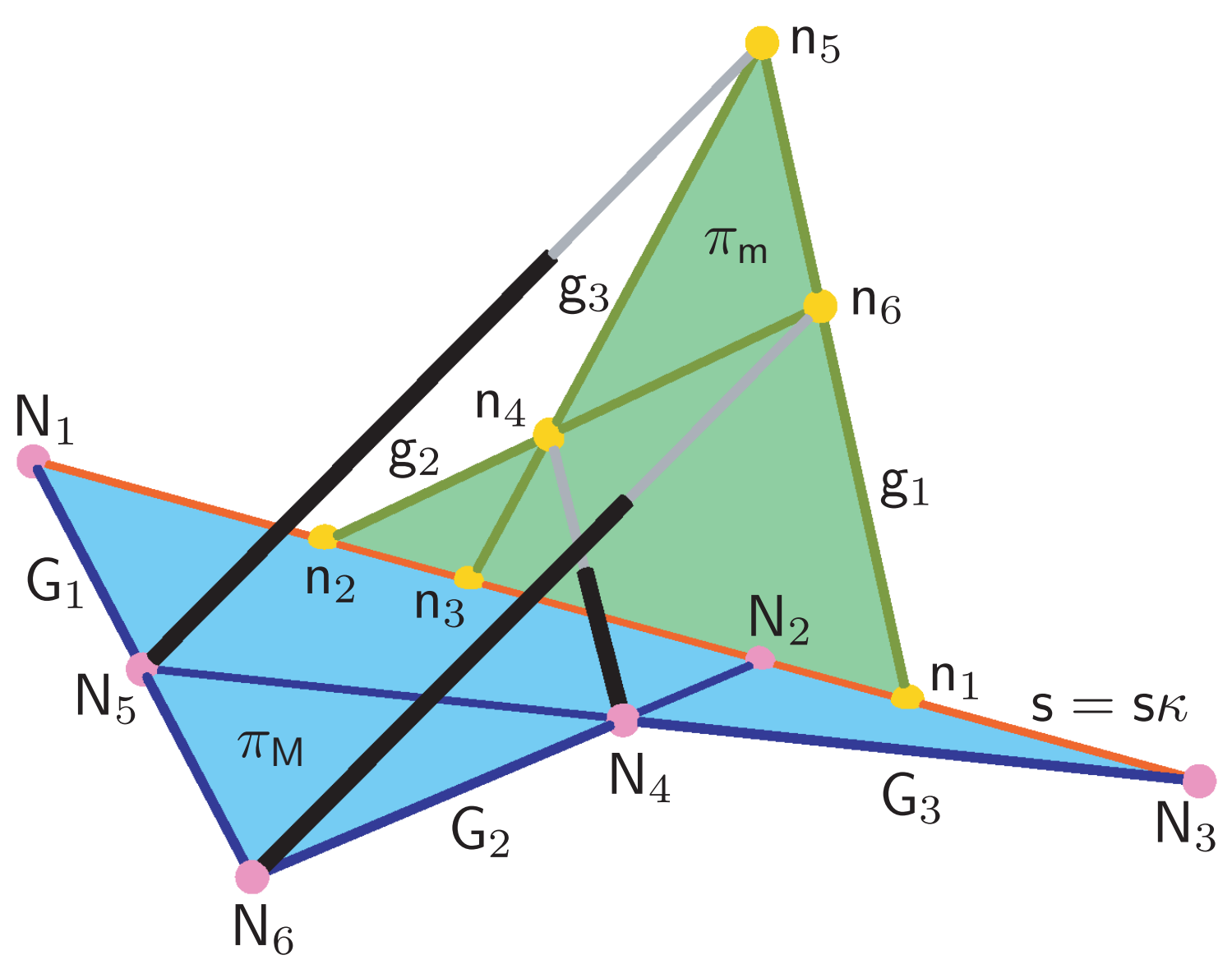

Due to Lemma 1 and the results of Borras et al. [11], we can replace the original six legs $\overline{\mathrm{m}_{i} \mathrm{M}_{i}}$ with $i=1, \ldots, 6$ by a new set of six legs $\overline{\mathrm{n}_{i} \mathrm{~N}_{i}}$ without changing the direct kinematics and singularity surface, if $\mathrm{n}_{i} \kappa=\mathrm{N}_{i}$ holds and $\mathrm{n}_{1}, \ldots, \mathrm{n}_{6}$ are not located on a conic section. 


\section{Proof of the Conjecture}

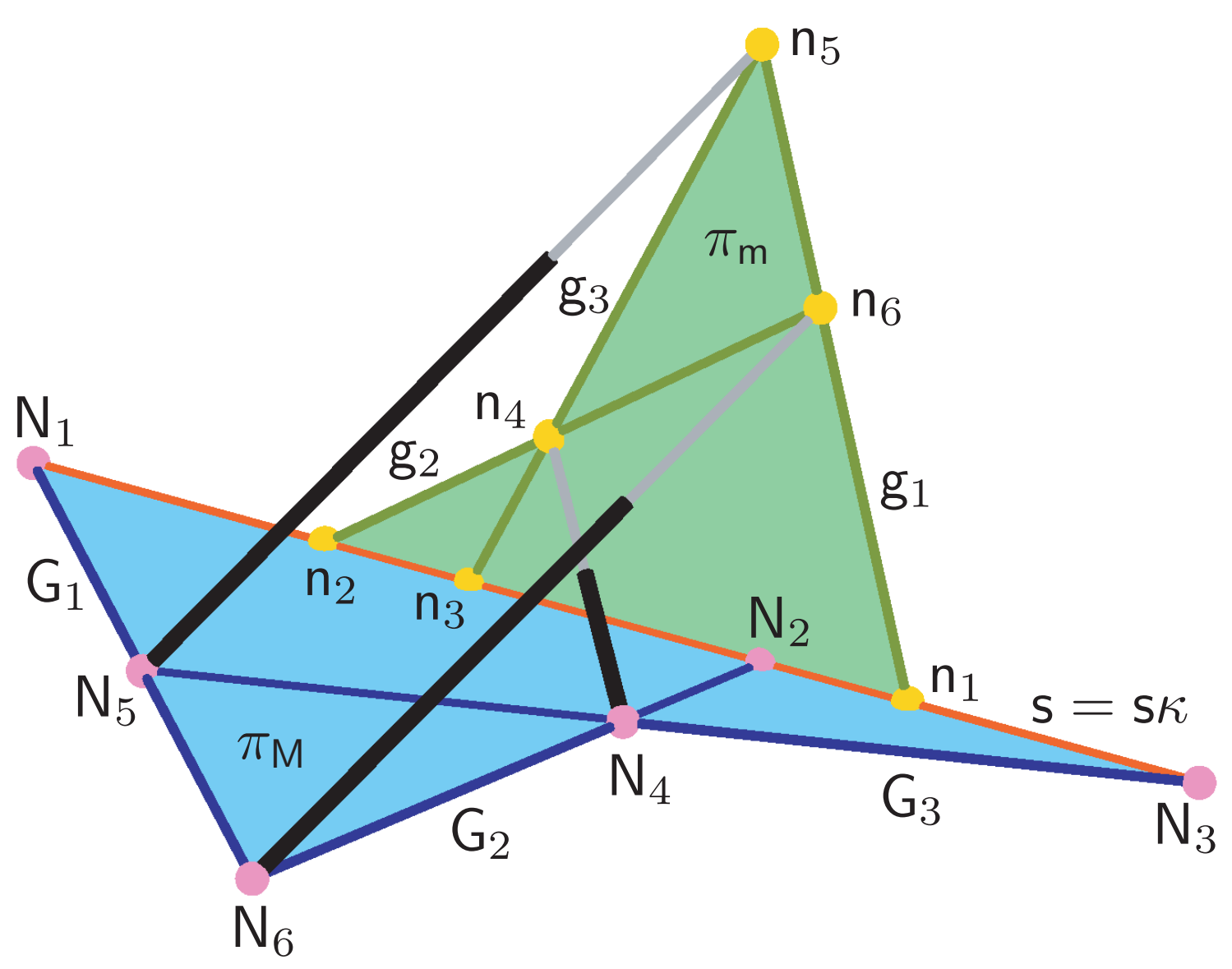

Due to Lemma 1 (Theorem 5), we can add the one-parametric set of legs $\overline{\mathrm{nN}}$ with $\mathrm{n} \in \mathrm{g}_{1}$, $\mathrm{N} \in \mathrm{G}_{1}$ and $\mathrm{n} \kappa=\mathrm{N}$ without disturbing the elliptic self-motion.

The lines $g_{1}$ and $G_{1}$ are skew $\left(\Leftrightarrow \mathrm{n}_{1} \neq \mathrm{N}_{1}\right.$ ), as the projectivity of $\mathrm{s}$ onto itself is elliptic. 


\section{Proof of the Conjecture}

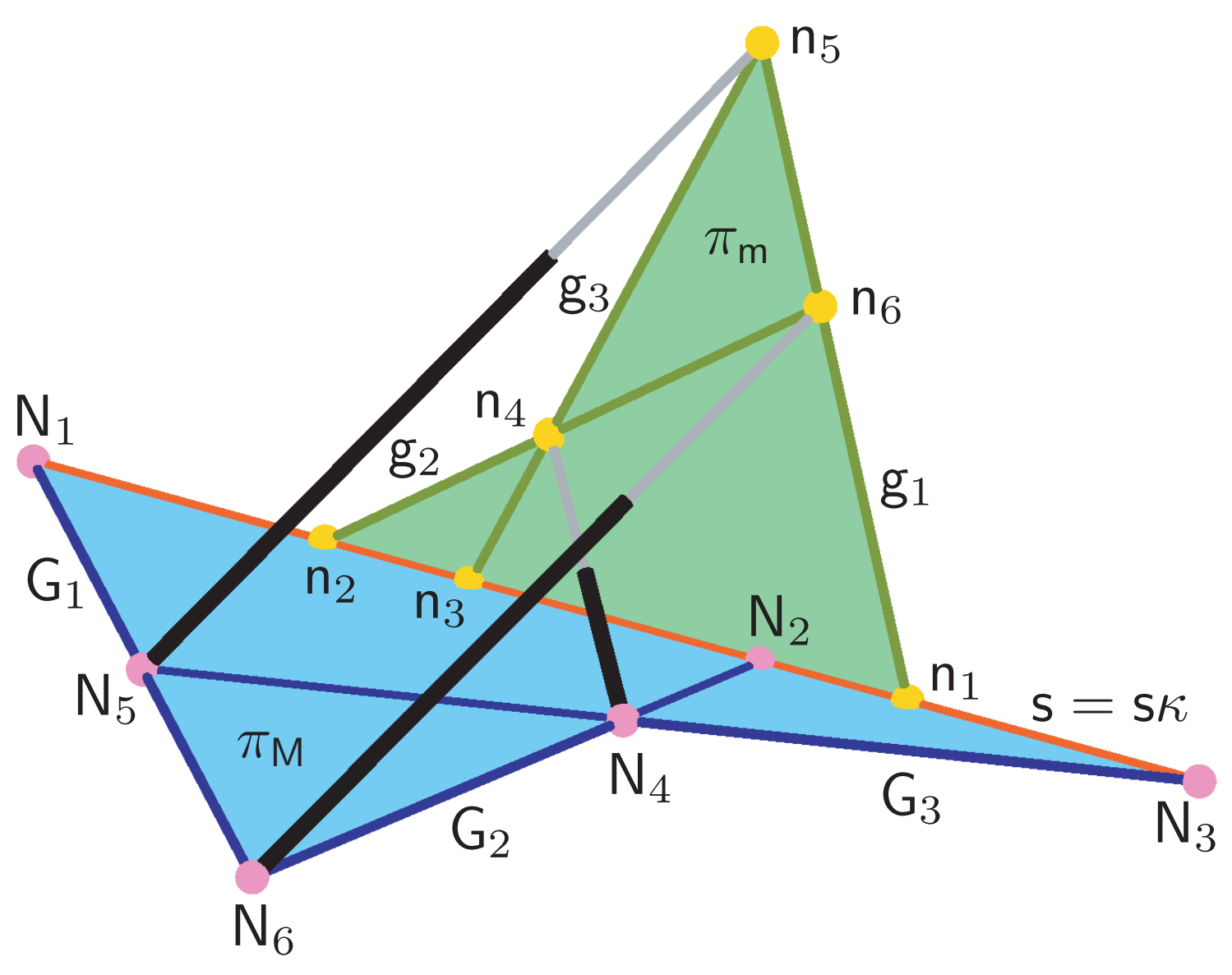

Therefore the one-parametric set $\mathcal{R}_{1}$ of lines $[\mathrm{n}, \mathrm{N}]$ is a regulus of a regular ruled quadric $\Phi_{1}$.

Due to the results of Henrici and Schur, we can add even arbitrary lines of the associated regulus $\mathcal{R}_{1}^{\times}$without restricting the elliptic self-motion. 


\section{Proof of the Conjecture}

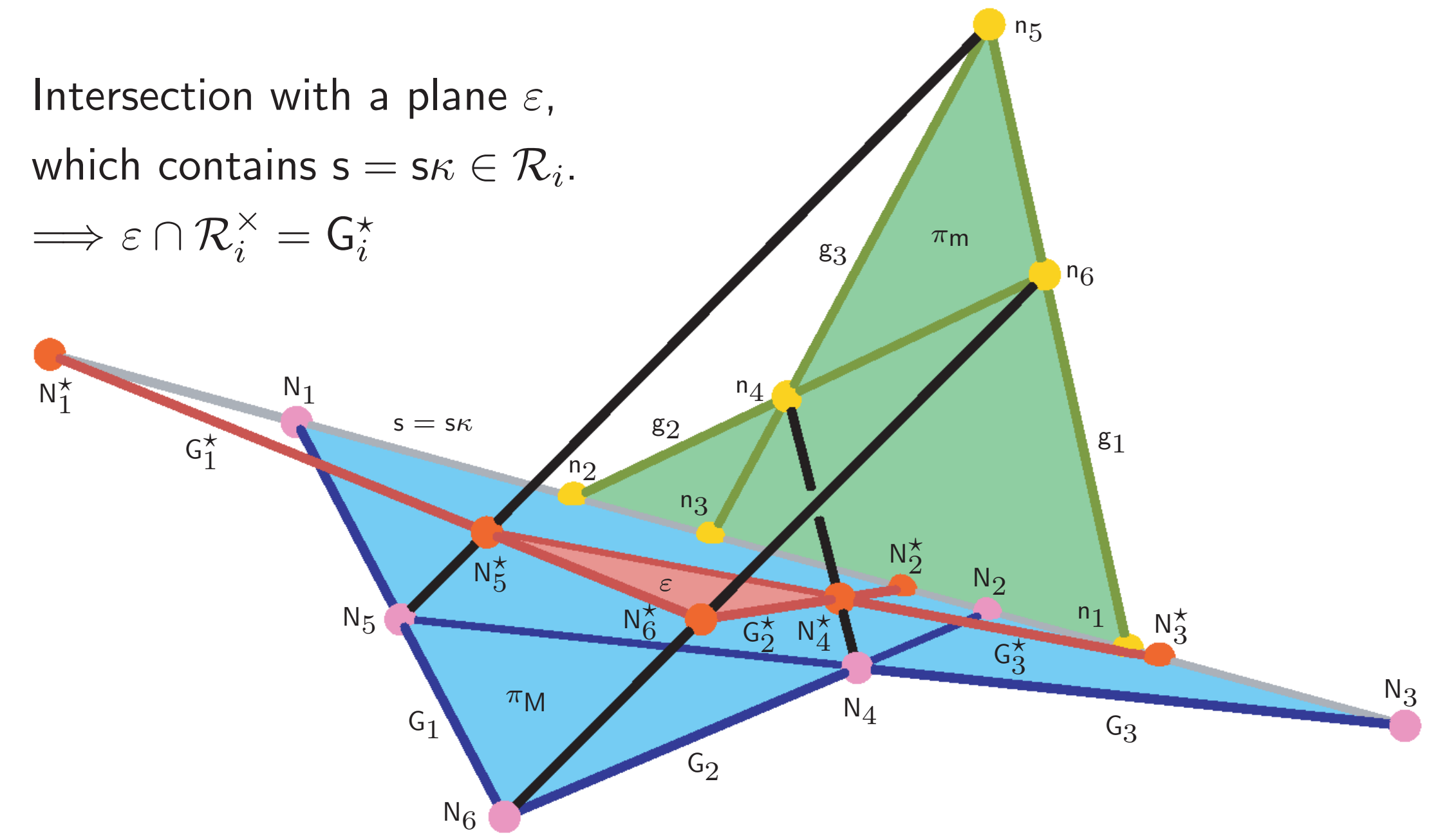




\section{Proof of the Conjecture}

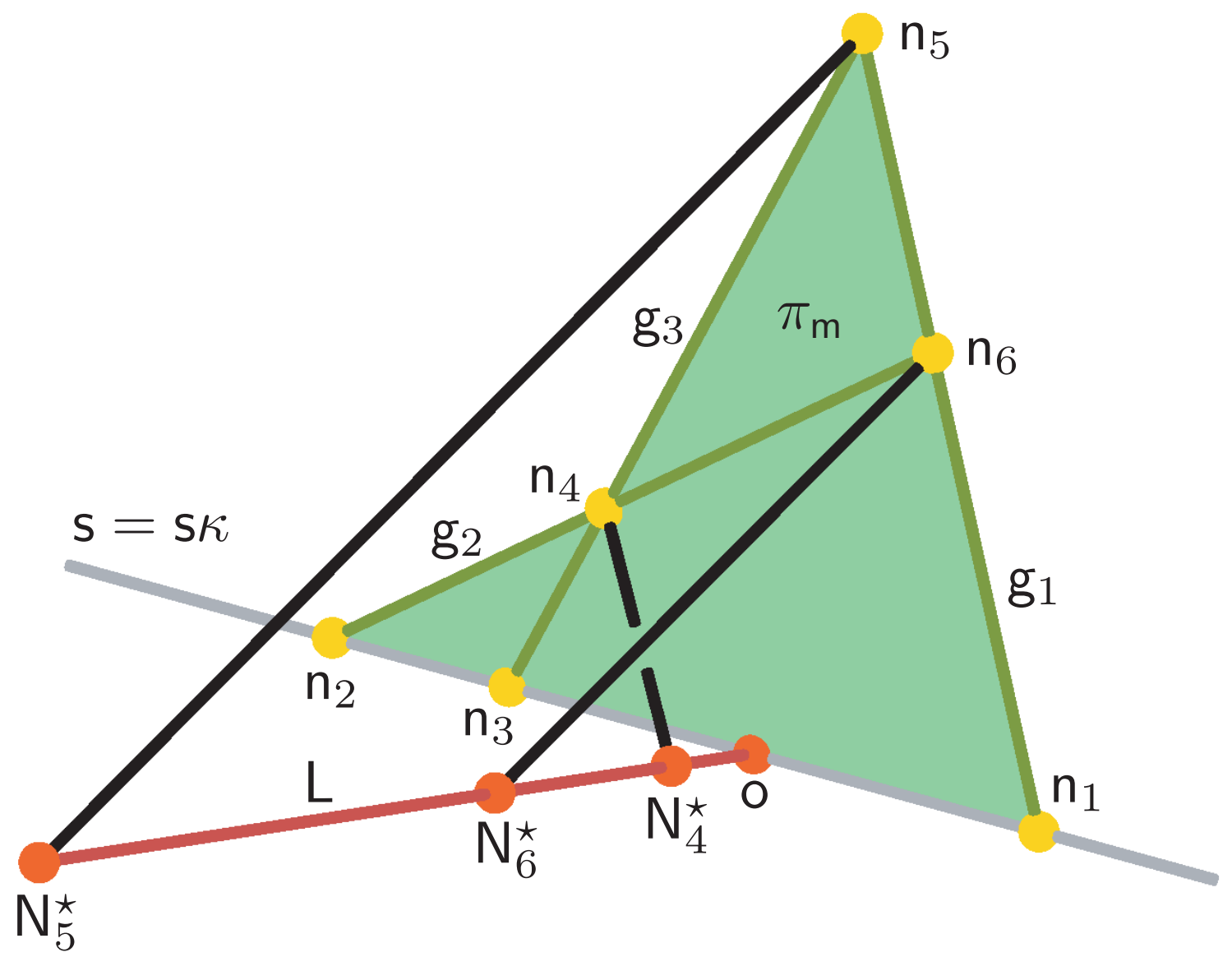

\section{Lemma 2}

The points $N_{4}^{\star}, N_{5}^{\star}, N_{6}^{\star}$ are pairwise distinct and do not belong to $\mathrm{s}=\mathrm{s} \kappa$. Moreover $\mathrm{N}_{4}^{\star}, \mathrm{N}_{5}^{\star}, \mathrm{N}_{6}^{\star}$ are not collinear.

\section{Proof of Lemma 2}

See the presented paper. 


\section{Proof of the Conjecture}

\section{Lemma 3}

There exists a non-singular projectivity $\kappa^{\star}$ with $\mathrm{n}_{i} \kappa^{\star}=\mathbf{N}_{i}^{\star}$ for $i=1, \ldots, 6$. Therefore the manipulator with platform anchor points $\mathrm{n}_{1}, \ldots, \mathrm{n}_{6}$ and base anchor points $\mathrm{N}_{1}^{\star}, \ldots, \mathrm{N}_{6}^{\star}$ is also a planar projective SGP with an elliptic self-motion.

\section{Proof of Lemma 3}

Due to Lemma 2, the points $\mathrm{N}_{1}^{\star}, \mathrm{N}_{2}^{\star}, \mathrm{N}_{4}^{\star}, \mathrm{N}_{5}^{\star}$ always form a quadrangle. Therefore the mapping $\mathrm{n}_{i} \mapsto \mathbf{N}_{i}^{\star}$ for $i=1,2,4,5$ uniquely defines a regular projectivity $\kappa^{\star}$, which also yields $\mathrm{n}_{3} \kappa^{\star}=\mathrm{N}_{3}^{\star}$ and $\mathrm{n}_{6} \kappa^{\star}=\mathrm{N}_{6}^{\star}$.

The elliptic self-motion of the manipulator $\mathrm{n}_{1}, \ldots, \mathrm{N}_{6}$ is transmitted by the motion of the reguli $\mathcal{R}_{1}, \mathcal{R}_{2}, \mathcal{R}_{3}$ onto the manipulator $\mathrm{n}_{1}, \ldots, \mathrm{N}_{6}^{\star}$.

This resulting self-motion is elliptic too, as a fixed point of the restriction of $\kappa^{\star}$ on $\mathrm{s}=\mathrm{s} \kappa^{\star}$ also has to be a fixed point of the restriction of $\kappa$ on $\mathrm{s}=\mathrm{s} \kappa$. 


\section{Proof of the Conjecture}

Construction for a special choice of $\varepsilon$ :

$S \ldots$ finite point of $s=s \kappa$

$\alpha \ldots$ plane spanned by $[\mathrm{S}, \mathrm{f}, \mathrm{F}]$

$\beta$... plane orthogonal to $\mathrm{f}$ through $\mathrm{S}$

$\mathrm{t} \ldots$ intersection line of $\alpha$ and $\beta$

$\varepsilon$...plane spanned by $\mathrm{t}$ and $\mathrm{s}=\mathrm{s} \kappa$

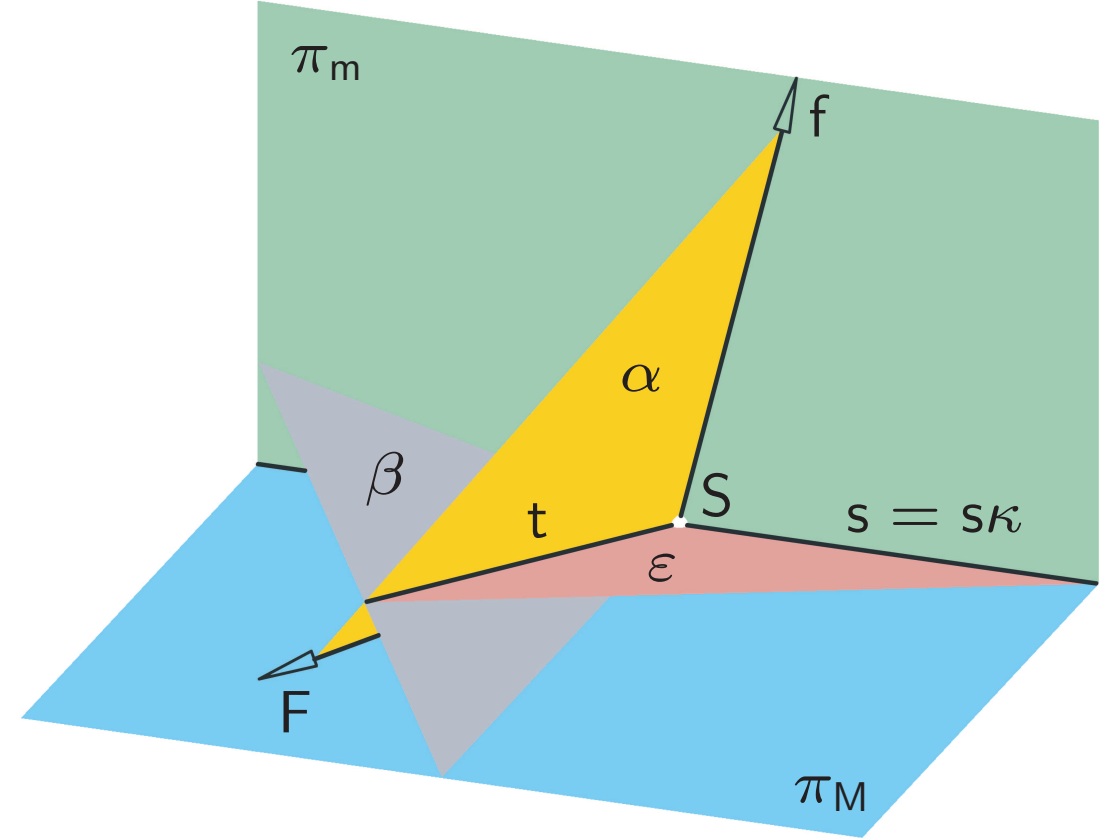

As $f \kappa^{\star}$ equals the ideal point of $t$, the self-motion of the planar projective SGP $\mathrm{n}_{1}, \ldots, \mathrm{N}_{6}^{\star}$ is orthogonal.

Therefore Theorem 3 yields the contradiction, which proves the conjecture. 


\section{Conclusion}

As the proof of the conjecture also closes the study of planar projective SGPs with self-motions, we can give the following main theorem under consideration of the results achieved in [5]:

\section{Theorem 6}

A planar projective SGP, which is not architecturally singular, can only have a self-motion, if the projectivity is an affinity $\mathbf{M}_{i}=\mathbf{a}+\mathbf{A} \mathbf{m}_{i}$, where the singular values $s_{1}$ and $s_{2}$ of the $2 \times 2$ transformation matrix $\mathbf{A}$ with $0<s_{1} \leq s_{2}$ fulfill the condition $s_{1} \leq 1 \leq s_{2}$.

All one-parametric self-motions are circular translations. Moreover the self-motion is a two-dimensional translation, if and only if, the platform and the base are congruent and all legs have equal length. 


\section{References}

[1] Merlet JP (1989) Singular configurations of parallel manipulators and Grassmann geometry, International Journal of Robotics Research 8(5):45-56

[2] Chasles M (1861) Sur les six droites qui peuvent étre les directions de six forces en équilibre, Comptes Rendus des Séances de l'Académie des Sciences 52:1094-1104

[3] Röschel O, Mick S (1998) Characterisation of architecturally shaky platforms, In: Lenarcic J, Husty ML (eds) Advances in Robot Kinematics: Analysis and Control, Kluwer, pp 465-474

[4] Karger A (2002) Singularities and self-motions of a special type of platforms, In: Lenarcic J, Thomas F (eds) Advances in Robot Kinematics: Theory and Applications, Springer, pp 155-164

[5] Nawratil G (2012) Self-motions of planar projective Stewart Gough platforms, In: Lenarcic J, Husty M (eds) Latest Advances in Robot Kinematics, Springer, pp 27-34

[6] Nawratil G (2013) On elliptic self-motions of planar projective Stewart Gough platforms, Transactions of the Canadian Society for Mechanical Engineering 37(1), in press

[7] Dycks W (1892) Katalog mathematischer Modelle, Wolf, München

[8] Schur F (1899) Die Deformation einer geradlinigen Fläche zweiten Grades ohne Änderung der Längen ihrer Geraden, Zeitschrift für Mathematik und Physik 44:62-64

[9] Wiener H (1907) Abhandlungen zur Sammlung mathematischer Modelle, Teubner, Leipzig

[10] Koenigs G (1897) Leçons de Cinématique, Hermann, Paris

[11] Borras J, Thomas F, Torras C (2010) Singularity-invariant leg rearrangements in doubly-planar Stewart-Gough platforms, Proc. of Robotics Science and Systems, Zaragoza, Spain 